\title{
Application of Fenestrated Aneurysm Clip for Incomplete Clamping of the Common Carotid Artery During Carotid Endarterectomy
}

\author{
-Technical Note- \\ Masakazu KOBAYASHI, Kuniaki OgASAWARA, Kenji YOSHIDA,
Yasunari OTAWARA, and Akira OGAWA
}

Department of Neurosurgery, Iwate Medical University, Morioka, Iwate

\begin{abstract}
Complete occlusion of the common carotid artery with blood vessel forceps may be prevented by hard atherosclerotic plaque during carotid endarterectomy. Incomplete clamping can be recognized via insertion of an elastic catheter, connected to a pressure transducer, into the common carotid artery. Application of a straight fenestrated clip parallel to the clamp avoids the plaque and occludes the vessel more distal to the fulcrum of the clamp, achieving complete arterial occlusion without applying excessive closing force on the artery.
\end{abstract}

Key words: carotid endarterectomy, common carotid artery clamping, fenestrated aneurysm clip

\section{Introduction}

Various types of blood vessel forceps (e.g., bull dog clamp, Fogarty clamp, Satinsky Atauma Blood Vessel Forceps (SNAA Industries, Punjab) can be used to clamp the common carotid artery during carotid endarterectomy. ${ }^{1-5)}$ However, the presence of hard atherosclerotic plaque can prevent complete occlusion of the artery. ${ }^{1)}$ In this case, the closing force of the blood vessel forceps is increased, or another forceps of the same type can be applied to achieve complete occlusion of the artery. However, these procedures may lead to plaque rupture or arterial wall injury. ${ }^{1,2)}$

Here we describe the application of a fenestrated aneurysm clip in parallel with the blood vessel forceps to achieve complete occlusion of the common carotid artery during carotid endarterectomy.

\section{Operative Technique}

Firstly, for clamping of the common carotid artery, we select a portion in the artery that is considered as absence of an atheroma plaque in preoperative cervical angiography and echography. Before clamping

Received May 19, 2008; Accepted December 26, 2008 of the carotid arteries, a 22-gauge elastic catheter is inserted into the common carotid artery and connected to a pressure transducer. The degree of carotid artery occlusion is determined with the pressure transducer. If incomplete clamping of the common carotid artery with a blood vessel forceps is recognized, a straight fenestrated clip (Sugita ${ }^{\mathbb{R}}$ Aneurysm Clip No. 29; Mizuho Ikakogyo Co., Ltd., Tokyo) is applied in parallel to the forceps (Fig. 1).

This technique was applied in 29 patients with incomplete clamping of the common carotid artery after positioning of the blood vessel forceps. Complete occlusion of the artery without intraoperative cerebral embolism or arterial wall injury was achieved in all 29 patients.

\section{Discussion}

The presence of hard atherosclerotic plaque near the fulcrum of the blood vessel forceps prevents complete arterial occlusion, even if a second set of forceps is applied in the same manner (Fig. 2). In contrast, application of a straight fenestrated clip parallel to the clamp avoids the plaque and occludes the vessel more distal to the fulcrum of the clamp. Therefore, the present technique results in complete arterial occlusion without excessive increase in the closing force. In addition, while closing force in the 


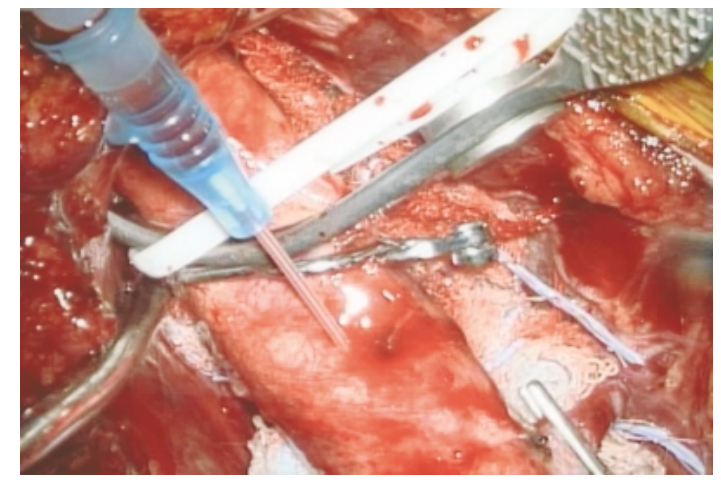

Fig. 1 Intraoperative photograph showing a straight fenestrated clip (arrow) applied from the same direction as the previously positioned bull dog clamp and parallel to the vessel clamp.
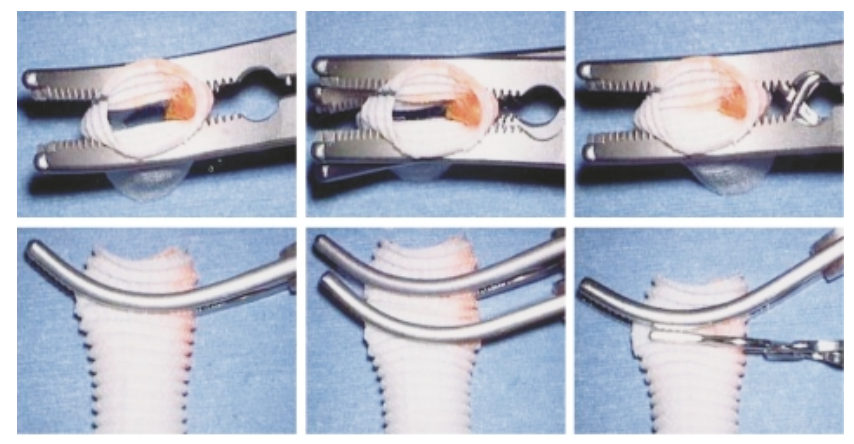

Fig. 2 Experimental model illustrating application of a fenestrated aneurysm clip for incomplete clamping of the common carotid artery. A cotton bar pasted in the inner wall of a gelatin impregnated knitted vascular prosthesis (Gelsoft ${ }^{\text {TM }}$ Plus; Sulzer Vascutek Ltd., Renfrewshire, Scotland, U.K.) is used to represent the hard eccentric atherosclerotic plaque. The tips of the bull dog clamp applied to the vessel from the side of the hard atherosclerotic plaque are unable to approximate, and the vessel remains incompletely occluded (left column). Application of an additional bull dog clamp from the same direction cannot correct this situation (center column). However, application of a straight fenestrated clip parallel to the clamp avoids the plaque and occludes the vessel more distal to the fulcrum of the clamp, achieving total vessel occlusion (right column). Upper and lower rows indicate top-bottom and lateral views, respectively. blades of the blood vessel forceps is weaker in the portion proximal to the fulcrum than in the portion distal to the fulcrum, that of the straight fenestrated clip may be approximately constant in any portion. Thus, this technique may be equally effective for both concentric and eccentric atherosclerotic plaque. Finally, the straight fenestrated clip is readily available in the neurosurgical operating room.

The present technique possesses one limitation. When there is a hard atherosclerotic plaque in the vessel wall more distal to the fulcrum of the clamp, the present technique may be less effective. In that case, a simple straight clip should be applied in parallel to the forceps and only the vessel wall near the fulcrum of the blood vessel forceps should be occluded.

\section{References}

1) Endo S, Hayashi N, Hirashima Y: [Surgical anatomy and techniques of carotid endarterectomy without shunt]. No Shinkei Geka 29: 605-615, 2001 (Jpn)

2) Findlay JM, Lougheed WM: Carotid microendarterectomy. Neurosurgery 32: 792-798, 1993

3) Little NS, Meyer FB: Carotid endarterectomy: indications, techniques, and Mayo Clinic experience. Neurol Med Chir (Tokyo) 37: 227-235, 1997

4) Loftus CM: Carotid Endarterectomy: Principles and Technique. St Louis, Quality Medical Publishing, 1995, pp 1-242

5) Spetzler RF, Martin N, Hadley MN, Thompson RA, Wilkinson E, Raudzens PA: Microsurgical endarterectomy under barbiturate protection: a prospective study. J Neurosurg 65: 63-73, 1986

Address reprint requests to: Kuniaki Ogasawara, M.D., Department of Neurosurgery, Iwate Medical University, 19-1 Uchimaru, Morioka, Iwate 020-8505, Japan.

e-mail: kuogasa@iwate-med.ac.jp

\section{Commentary}

Carotid endarterectomy is one of the few operations performed by neurosurgeons that has been demonstrated to be of benefit on the basis of randomized clinical trials. The demonstrated benefit of this procedure over other therapeutic options or natural history presumes a very low complication rate. Successful and durable carotid endarterectomy is dependent on meticulous attention to detail. Kobayashi and colleagues have developed and shared a clever technique for occluding the common carotid artery in circumstances where traditional forceps fail to occlude the 
artery due to intraluminal plaque. This technique provides a safe and atraumatic option for temporary common carotid artery occlusion during carotid endarterectomy.

Daniel L. BARRow, M.D. Department of Neurosurgery Emory University Atlanta, Georgia, U.S.A.

In this brief technical note, the authors described a method to solve the problem of incomplete occlusion of the common carotid artery during cross clamping of the artery in carotid endarterectomy. Under the condition that a thick atherosclerotic plaque of the common carotid artery existing at the site of clamping to prohibit complete occlusion of the artery by the clamp, a second straight fenestrated aneurysm clip placed parallel to the clamp may encircle the area with plaque and compress the rest of the arterial lumen to achieve a complete occlusion. In our own practice, we also use the similar technique to clip large or giant aneurysm with partial atherosclerotic change on the aneurysm to achieve complete obliteration of the aneurysmal neck.

However, in carotid endarterectomy if a thick atherosclerotic plaque is found at the site of cross clamping, this plaque should be removed and endarterectomy should be extended to this area. Thus, the condition described by the authors is a condition rarely existing in our daily practice.

Due to the worry of bleeding from incomplete occlusion, the authors inserted a 22-gauge catheter into the common carotid artery to monitor the intraluminal pressure to detect incomplete occlusion of the artery. This procedure may cause dislodge of the thrombus and plaque to increase the surgical risk. Bleeding from incomplete occlusion of the common carotid artery after arteriotomy can be controlled if a tourniquet as an additional temporary proximal control is placed around the artery before arteriotomy.

Yong-Kwang TU, M.D. Department of Neurosurgery College of Medicine and Hospitals National Taiwan University Taipei, Taiwan 